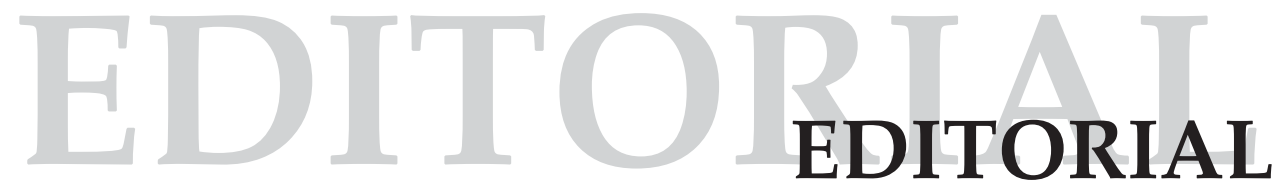

\title{
Territorialidades rururbanas o rururbanidad contemporánea
}

La rururbanidad constituye una nueva forma de ciudadanía en derecho. En esa medida, lo(el) rurubano es una de las figuras territoriales más potentes; a tal grado de haber conformado hoy en día, y desde hace más de tres décadas, un "tercer territorio": nuevas o renovadas morfologías y dinámicas de geograficidad, sociabilidad e historicidad.

En la América global y Europa, este tercer territorio llega sin que hayamos resuelto la relación y delimitación histórica del campo y la ciudad. En Europa, la saldaron con el discurso dominante que desde hace cuatro décadas convirtió a los campesinos en rurales y con ello la diferenciación gubernamental quedó hecha. No obstante, en mis últimas investigaciones en el Sur de Francia, he podido constatar que la diferencia en la vida cotidiana es tan marcada como antes. En el trabajo de campo realizado, en junio de 2017, una mujer entrevistada que habita esa zona desde hace 30 años — producto de la ola neo-rural - me describió un incidente con un antiguo vecino; hecho que la hizo poner en consciencia de que ella era rural y él campesino (Nates-Cruz, 2017). Me relató que un día al pasear en bicicleta con su perro por los alrededores de las fincas donde ambos vivían, el perro comenzó a ladrar de manera desproporcionada al hombre y entonces él reaccionó "como un campesino"; en sus palabras:

[...] tomó un bastón y corriendo lo golpeo [...]. Me enojé mucho y lo inquirí fuertemente y él peor. Pero al final debimos conversar y buscar una manera de hacer las paces aunque fuera sólo una forma de cortesía porque vivíamos casi [uno sobre otro]. 
Yo le pregunté, si se había dado cuenta del contenido de la descripción que me estaba dando; a lo que, interpelada, me respondió: "es verdad, vivimos en el campo, pero él es campesino y yo soy rural". Pero no solo eso, ya que la escena también deja ver la imagen bucólica de una urbanita paseando en bicicleta con su perro y de un hombre de campo con actitudes, para ella, agrestes puesto que él no entendía cómo un perro paseaba y ladraba sin respeto al nativo; es más, cómo un perro paseaba, si, para él, en el campo, los perros están al cuidado de las casas. Los hechos aquí descritos marcan dos civilidades que se encuentran en esos lugares rurales (o rururbanos).

Consideramos que no es débil pensar que una figura territorial como estas, esté en germen desarrollándose en el campo. Que las civilidades u urbanidades que generan formas de ciudadanía distinta a la propiamente urbana o propiamente rural, están teniendo lugar. Estas formas de saber estar cuentan en su tiempo-espacio y dejan ver las nuevas civilidades que se están construyendo; en donde ese tercer territorio emerge cada vez con más fuerza, dejando a menudo marginadas a las poblaciones nativas o instalando nuevas formas y medios de saber estar. Bien tiene razón Cardoso (2012) cuando plantea que de fenómeno ha pasado a ser un problema territorial y cuando, aún más, al contrario de algunos autores (Ávila, 2009; Arias 2005), se sostiene aquí que las áreas rururbanas ubicadas dentro y fuera de las franjas que colindan entre campo-ciudad no son en América Latina -y tampoco lo son en estudios demostrados para el caso del Sur de Francia y el Norte de España- (Nates-Cruz, 2007, 2011, 2017) tierras baldías e improductivas; por el contrario se están interviniendo zonas de potencial desarrollo agrario campesino-rural para transformarlas en áreas residenciales, agroindustriales y de otros sectores socioeconómicos y sin ningún componente de equidad territorial inmediato.

En ningún caso, pero en particular en Colombia, lo rururbano debe ser visto como áreas de transición. Estas se presentan como complejo de lugares con dinámicas propias que no solo exigen correlaciones entre nativos rurales y urbanitas que conforman la población rururbana (cuando no hay 'vaciado' de población nativa como sucede a menudo) sino también correlación entre urbanitas de distintas procedencias, cultura, economía y educación. En el caso de los urbanitas ese germen de nueva forma de ciudadanía exige ejercicios tan simples como, por ejemplo, no poder votar en la ciudad sino en la vereda o cabecera municipal a la que pertenece el lugar que habitan. El desequilibrio del 'propio' con relación al 'foráneo' se hace sentir y lo hay en múltiples casos en ciudades como Manizales, Bogotá, Medellín, Cali, Pereira, Ibagué o Popayán — por poner solo algunos ejemplos-; en donde considerables extensiones de lo rururbano se han 
construido con el 'vaciado' de una población campesina nativa que vivía en las franjas rural-urbanas de dichas ciudades. La compra de tierras por parte de particulares o de empresas inmobiliarias ha sido el primer síntoma local de este tipo de fenómeno; tema del que se ocupan varios autores (NatesCruz, 2008, 2014; Zuluaga, 2008; Acosta Nates, 2017; Sánchez Torres, 2017).

Han sido considerables y potentes las denominaciones de los impulsos o causas de conformar lo rururbano y la rururbanidad, así como de las denominaciones del asentamiento territorial mismo. Son variadas y cada una no solo enfatizando en una dimensión territorial sino también en una dimensión antropológica que toca la identidad, el sentido de pertenencia y la organización social.

En el primer grupo de denominaciones debemos situar la búsqueda de la naturaleza, el retorno al campo y el retorno a la tierra como figuras que han marcado la causa o impulso de conformar lo rururbano. Estas figuras son categorías analíticas y de vivencia empírica que por tradición científica se han asignado a procesos que se viven en Europa, Estados Unidos y Canadá (Mallet, 1941; Hervieu, 1979; Keyser, 1983; Gavignaud, 1990; Andreani, 1999; Bradshaw, 1993; Poulle and Gorgeu, 1997; Urbain, 2002; Perrier-Cornet, 2002; Raymond, 2003; Nates-Cruz y Raymond, 2007). Poco se ha dicho sobre esos procesos en América Latina. Las dinámicas territoriales que se han configurado en países como Colombia, México, Argentina, Brasil, Perú, Ecuador (Pérez, 2001; Hierneaux, 2001; Arias, 2002; Ávila, 2005; Zuluaga, 2005; González, 2008; Nates-Cruz, 2008; Malizia, 2011; Llambí, 2012; Urzúa, 2013; Macuacé, 2014; Acosta Nates, 2016), muestran desde avanzados los años 80 que estas prácticas de buscar la naturaleza y retornar al campo o retornar a la tierra se han instalado en total equivalencia epistemológica con otras realidades del mundo. Esta cercanía se objetiva, no obstante, de manera distinta a través de su ontogénesis.

En el segundo grupo encontramos categorías (naturalezas socioespaciales) tales como ciudades rurales, pueblos urbanos (Licona, 2016), barrios rurales (Nates-Cruz, 2014); en todo caso, un rururbano, que viene generando en América Latina verdaderas villas rurales con una coexistencia temporal de narrativas distintas y simultaneidad de trayectorias contemporáneas como característica inherente de ese tercer territorio. Este 'traslado' hacia las zonas rurales se hace muy de la mano de las inmobiliarias que construyen mundos aparte para que los habitantes tengan la idea de que siguen en la ciudad con un imaginario de campo. Y el consumo en toda su expresión es la característica fundamental. Debemos decir que en países como México, Colombia, y ahora en Argentina, la influencia del capital económico del narcotráfico también ha contribuido a la conformación acelerada del rururbano residencial. En el tema, sobre 
todo, es la prensa nacional (Clarín, La Nación, El Espectador, El Tiempo, Reforma, La Jornada) la que monitorea el caso. Se hace difícil lograr estadísticas oficiales. Sin embargo buenas inferencias se pueden hacer si se examinan los bienes incautados a las inversiones residenciales en medio rural - tanto en forma de villas, parcelaciones y equipamientos como de residencias de corte sectorial no rurales- que 'decoran' de manera desproporcionada el campo desde usos y manejos urbanos. Datos como los que presenta la Sociedad de Activos Especiales - SAE_, entidad que administra los bienes inmuebles de la Dirección Nacional de Estupefacientes —DNE— en Colombia, mostró en agosto de 2013 que había cerca de 200 predios e inmuebles en zonas rurales y urbanas.

Por último, en Colombia, se ha demostrado que ese "tercer territorio" que es lo rururbano a acaecido sin que se haya proyectado legislar sobre su ordenamiento territorial más que en torno a lo que se denomina vivienda campestre. La Ley Orgánica de Ordenamiento Territorial 1454, que data de 2011, es una ley funcional que se creó más como un marco de apoyo a la gestión técnica territorial que a una ordenación territorial pensada desde todo el compendio requerido para un país que como este ha vivido más de 50 años en guerra civil; en cuyo proceso las categorías campo-ciudad hace mucho tiempo se movieron del mapa con todas sus consecuencias. En el tema que nos ocupa lo que más se acerca en la reglamentación, pensando desde lo urbano como expansión de lo rural, es cuando se hace alusión en la mencionada ley a las competencias de los municipios: "reglamentar de manera específica los usos del suelo, en las áreas urbanas, de expansión y rurales, de acuerdo con las leyes".

Sin embargo las evidencias de un rururbano sin control son cada vez más numerosas, en particular en la Región Andina del país. Aquí encontramos en grandes ciudades como Medellín, Bogotá y Cali, o en ciudades de menor talla en extensión y población como Pereira, Manizales, Villavicencio, Popayán y Bucaramanga, que la expansión urbana a las áreas rurales y la intervención de estas más allá de la franja física campo-ciudad es pronunciada. Este es un país moderno a nivel discursivo-normativo, pero tiende a la premodernidad en cuestión de la relación práctica-norma. Lo que nos rige, por ejemplo, desde la ley que desarrolla los asuntos de áreas metropolitanas que tocan el contacto rural-urbano-ambiental (Ley 388 de 1997, artículos 7 y 15) da pistas para una mejor consciencia territorial en términos de manejo y gestión rururbana sin que sea a menudo tenida en cuenta:

la expedición de normas para la parcelación de predios rurales destinados a vivienda campestre [deben] tener en cuenta la legislación agraria y ambiental. 
[...] las normas urbanísticas regulan el uso, la ocupación y el aprovechamiento del suelo y definen la naturaleza y las consecuencias de las actuaciones urbanísticas indispensables para la administración de estos procesos. Estas normas estarán jerarquizadas de acuerdo con los criterios de prevalencia aquí especificados y en su contenido quedarán establecidos los procedimientos para su revisión, ajuste o modificación [...] los municipios deberán formular y adoptar los planes de ordenamiento del territorio [también lo pide la LOOT de 1454], reglamentar de manera específica los usos del suelo en las áreas urbanas, de expansión y rurales de acuerdo con las leyes, optimizar los usos de las tierras disponibles y coordinar los planes sectoriales, en armonía con las políticas nacionales y los planes departamentales y metropolitanos.

El anterior marco de discusión convoca la temática de la revista en este número, denominada: "Rururbanidades contemporáneas".

Así, entonces, en esta oportunidad, el lector hallará en la Sección central o monográfica los siguientes artículos: “Abordajes teóricoconceptuales y elementos de reflexión sobre rururbanización desde los estudios territoriales", de Diana Sánchez, el cual se acerca analíticamente a modo de discusión a la definición de los conceptos de rururbanización y rururbanidades a partir de una revisión seria de la literatura relacionada. El segundo artículo, intitulado: "El binomio URBS/RUR como base de la concepción territorial y urbanística de Ildefonso Cerdà", de Joan Tort Donada y Albert Santasusagna Riu. Ambos investigadores nos proponen una mirada al pensamiento de Ildefonso Cerdà quien a través del significado atribuido a la urbe y a la urbanización, así como a la rur y a la rurización, constituiría una teoría general de la colonización del territorio y del urbanismo. El artículo "Ciencias sociales y sostenibilidad: tecnologías de investigación social aplicadas a lo urbano y lo rural", de Juan David Luján, logra mostrarnos la importancia de las tecnologías de investigación social para el abordaje de la problemática social en escenarios urbanos y rurales; insistiendo en lo cuantitativo y cualitativo, pero no necesariamente estadístico, y con énfasis en la actitud interdisciplinaria. Se cierra esta primera sesión con el artículo, "Metropolización versus ciudades intermedias. La incompatibilidad del auge de las ciudades intermedias en la fase actual de la urbanización de la población colombiana", del profesor e investigador Óscar Alfonso Roa. Artículo que discute la noción de ciudad intermedia, en contraste con el ámbito metropolitano, en términos de los efectos en los procesos de urbanización. 
En la sección Estudios de Región, aparecen: "Entre la pobreza y la producción agropecuaria: población rural dispersa en Colombia, 2016", de William Chará Ordoñez, Alexander Castillo Garcés y Juan Camilo López Martínez; los autores llevan a cabo un acercamiento al "mundo rural", zonas que han vivido condiciones de abandono y pobreza, e inquietarnos acerca de las posibilidades del fortalecimiento institucional con el fin de constituir una relación más productiva y de articulación entre el campo y las ciudades. Otro artículo de esta sección lo constituye: “Las prácticas sociales y la reincidencia de personas en proceso de reintegración, en el marco de la política nacional de reintegración económica y social", de Pamela Santa Montoya y Luis Adolfo Martínez Herrera, en el que reflexionan sobre las dinámicas de reincidencia de las prácticas sociales violentas en la población en proceso de reintegración; a pesar de que los autores no plantean hipótesis causales determinantes de ese fenómeno, si nos alertan sobre la necesidad de construir estrategias que permitan disminuir la reincidencia. La sección termina con el artículo de investigación: “Gramáticas sociales en los márgenes: formas de gobierno de la vida en el barrio Las Brisas de la ciudad de Pereira", de Óscar Jaramillo García, el cual analiza las gramáticas sociales como formas de gobierno a través de una visión local o barrial; constatando, en ese caso, la desciudadanización y el abandono del Estado en condiciones dramáticas que ponen en constante exposición la vida humana.

Para la sección Trayectos, finalmente, aparece el artículo de reflexión: "El pensamiento de Antonio Gramsci en América Latina y Colombia", de Rodrigo Santofimio Ortiz, que muestra a nivel latinoamericano la resonancia y apropiación del pensamiento del filósofo y marxista italiano. De igual forma sus efectos e implicaciones, en Colombia, en una coyuntura de alta virulencia social y política, admitiendo — tal y como lo muestra el autor- una recepción en el ámbito académico no exenta de discusiones y posturas crípticas.

Así pues, la Revista Antropología y Sociología: VIRAJES da la bienvenida a los lectores para que entren a estas nuevas realidades territoriales. Bienvenidos.

BÉATRIZ NATES-CRUZ Editora invitada Universidad de Caldas 


\section{Referencias bibliográficas}

Acosta Nates, P.A. (2016). Gentrificación rural: nuevos escenarios y economías emergentes. El caso de la vereda Clarete (Cauca, Colombia). En B. Nates-Cruz, Paisajes productivos y desarrollo económico territorial. Conflictos culturales, económicos y políticos. Manizales, Colombia: Universidad de Caldas.

Acosta Nates, P.A. (2017). Contextos e implicaciones residenciales generados por la gentrificación rururbana. Enfoque desde una antropología del territorio. Proyecto Doctoral. Universidad de Caldas, Manizales, Colombia.

Andreani, J.L. (7 de mayo de 1999). Quand les urbains rêvent de redevenir des ruraux. Le Monde, 19.

Arias, P. (2002). Hacia el espacio rural-urbano. Una revisión de la relación campo-ciudad en la antropología social mexicana. Estudios Demográficos y Urbanos, 17 (2), 363-381.

Ávila, H. (2005). Introducción. Líneas de investigación y el debate en los estudios urbanorurales. Lo urbano-rural ¿nuevas expresiones territoriales? Cuernavaca, México: CRIM, UNAM.

Bradshaw, T.K. (1993). In the Shadow of Urban Growth: Bifurcation in Rural California Communities. En A.T. Lyson and W.W. Falk, Forgotten Places: Poor Rural Regions in the United States (pp. 218-245). Kansas, USA: University Press of Kansas.

Cardoso, M. et al. (2012). Revisión de la definición del espacio rururbano y sus criterios de delimitación. Buenos Aires, Argentina: Contribuciones Científicas GÆA.

Gavignaud, G. (1990). Les campagnes en France au XXe siècle (1914-1989). Paris, France: Synthèse \& Histoire, Ophrys.

González, Plazas (2008). Estado actual de la Periurbanización y el hábitat periurbano en Manizales (Colombia). En: http://www.javeriana.edu.co/viviendayurbanismo/ pdfs/04CnosViv3.pdf

Hervieu, B. et Leger, D. (1979). Le retour à la nature: au fond de la forêt... l'Etat. Paris, France: Seuil.

Hierneaux, D. (2001). Las nuevas formas urbanas y reestructuración del mundo rural. En P. Torres (Comp.), Procesos metropolitanos y agricultura urbana. Xochimilco, México: Universidad Autónoma Metropolitana.

Kayser, B. (1983). Le village recomposé. Pour l'analyse du changement social et culturel en milieu rural. Toulouse, France: Université de Toulouse.

Licona, E. et al. (2016). San Miguel Canoa. Pueblo Urbano. Puebla, México: BUAP.

Llambí, L. (2012). Procesos de transformación de los territorios rurales latinoamericanos: los retos de la interdisciplinariedad. EUTOPÍA. Relación Campo Ciudad. Revista de Desarrollo Económico Territorial, 3, 117-134.

Macuacé, R.A. y Gómez Sánchez, A.M. (2014). Migración hacia los espacios rururbanos en Popayán (Colombia) para la primera década del siglo XXI. Revista de Economía del Caribe, 14. Recuperado de http://rcientificas.uninorte.edu.co/index.php/economia/article/view/6347/6567.

Malizia, M. (2011). Countries y barrios privados en el Gran San Miguel de Tucumán. Efectos y contrastes sociales (tesis de posgrado). Universidad Nacional de Tucumán, Tucumán, Argentina.

Mallet, R. (1941). Nécessités d'un retour à la terre (tesis de posgrado). Université de Paris, Paris, France.

Nates-Cruz, B. (2008). Productions territoriales et innovation des traditions des "natifs" face à l'implantation des Européens du Nord. Etude comparée en zones rurales française et espagnoles. En J.-P. Diry (Ed.), Les étrangers dans les campagnes. Clermont Ferrand, France: Presses Universitaires Blaise Pascal. 
Nates-Cruz, B. (2008). Procesos de gentrificación en lugares rururbanos: presupuestos conceptuales para su estudio en Colombia. Revista Antropología y Sociología: VIRAJES, 10, 253-269.

Nates-Cruz, B. (2014). De la política del lugar al lugar vivido: concepción y gestión territorial. En Conferencia en el SEMITEC-RETEC. Manuscrito.

Nates-Cruz, B. (2017). Revisite au territoire des anglais dix ans après. Productions territoriales et dynamiques socioculturelles a Saint Antonin Noble Val. Paris, France: Fondation Maison des Sciences de l'Homme et EHESS.

Nates-Cruz, B. y Raymond, S. (2007). Buscando la naturaleza. Migración y dinámicas rurales contemporáneas. Barcelona, España: Anthropos Editorial.

Pérez, M.E. (2001). Hacia una nueva visión de lo ruralidad. En N. Giarraca (Comp.), ¿Una nueva ruralidad en América Latina? Buenos Aires, Argentina: CLACSO.

Perrier-Cornet, P. (2002). Repenser les campagnes. Paris, France: Edition de l'Aube.

Poulle, F. et Gorgeu, Y. (1997). Essai sur l'urbanité rurale - Cinq territoires ruraux, leurs serments et leurs modes de gouvernement. Paris, France: Syros.

Raymond, S. (2003). Du "retour à la nature" au "retour à la champagne". Migrants et recompositions territoriales dans le Midi de la France et en Californie du Nord (tesis de posgrado). Université de Toulouse, Toulouse, France.

Sánchez Torres, D. (2017). Metamorfosis territorial para la objetivación de paisajes rururbanos desde procesos de gentrificación rural, resistencias e innovaciones campesinas. Estudio comparativo en Caldas, Risaralda y Quindío (1970-2018). Proyecto Doctoral. Universidad de Caldas, Manizales, Colombia.

Urbain, J-D. (2002). Paradis verts, désirs de campagnes et passions résidentielles. Paris, France: Edition Payot.

Urzúa, K.E. (2013). ¿Gentrificación contemporánea en contexto urbano-rural chileno?: el caso de los condominios cerrados de las comunas del Alto Aconcagua (tesis de posgrado). Universidad de Chile, Santiago de Chile, Chile.

Zuluaga, G.P. (2005). Dinámicas territoriales en frontera rural-urbana en corregimiento de Santa Elena, Medellín (tesis de posgrado). Universidad Nacional de Colombia, Medellín, Colombia. 